\title{
The job satisfaction-job performance relationship for creative tasks: An empirical investigation of the role of attitude and behavior in job performance among scientists
}

\author{
Naoko KATO-NITTA* \\ (Ibaraki University) \\ Tadahiko MAEDA** \\ (The Institute of Statistical Mathematics / \\ The Graduate University for Advanced Studies [Sokendai])
}

\begin{abstract}
This study examines whether job satisfaction-job performance relationship models vary when creativity considered as a task characteristic. Although previous studies have pointed out the possibility of a difference in the correlation strength between these two variables based on job complexity, the evidence is incomplete. Using data from a survey of Japanese scientists, we evaluated scientists' performance by categorizing their tasks into two types, creative tasks and formulaic tasks according to creativity demands, and used publication productivity as an objective indicator. We then investigated their relationships with job satisfaction. While the tasks differed in the level of creativity required, they both were high-complexity tasks. The results showed that a job satisfaction-job performance relationship was observed only for job performance related to creative tasks. Furthermore, we examined the need for considering the relationship at both individual and group levels. Our results were consistent for both levels.
\end{abstract}

Keywords : job satisfaction, job performance, creativity, scientists, public research institutions, university research administrator (URA)

\section{Introduction}

The relationship between job satisfaction and job performance has attracted the attention of many organizational researchers in recent decades. However, gaps in the research still remain. Contrary to the common intuitive assumption that happy workers who are satisfied with their jobs will work harder, previous studies have shown few consistent results regarding the correlation between job satisfaction and job

* Postdoctoral Researcher, Ibaraki University.

**The Institute of Statistical Mathematics / Associate Professor, The Graduate University for Advanced Studies [Sokendai]. performance. After Iaffaldano and Muchinsky (1985) conducted a meta-analysis and concluded that there was little correlation between the two, studies exploring this relationship temporarily declined. Judge et al. (2001) conducted a meta-analysis and reported a higher correlation between job satisfaction and job performance, presenting an average correlation coefficient of 0.30 . Encouraged by their findings, research interest in this area began to increase once again. While many empirical examinations exploring the relationship between job satisfaction and job performance have been conducted and have reported a correlation between the two variables, many researchers still hold the 
view that the correlation is spurious (e.g., Bowling, 2007). Considering the significance of the relationship between "the most focal attitude about the job (job satisfaction)" and "the most focal behavior on the job (job performance)" in the field of industrial/organizational psychology (Judge et al., 2001: 388), there is a need to re-examine this issue using an alternative approach. This study aims to deepen the understanding of the job satisfaction-job performance relationship by employing an approach from scientometrics for measuring publication productivity as a more objective indicator of job performance ${ }^{1}$.

Many researchers (e.g., Ivancevich 1979; Judge et al., 2001) have cited job complexity as a moderator between job satisfaction and job performance and found the correlation to be higher in high-complexity jobs than in lowcomplexity jobs. This hypothesis has indeed been substantially confirmed; however, some inconsistent evidence has been observed across occupations. Therefore, the current study proposes models that assume differences in task creativity but similarity in task complexity for job performance and examines how these models vary with regard to job satisfaction-job performance correlation strength. In particular, we focused on task differences among the same occupation, i.e., scientists engaged in research in the basic sciences in Japanese public research institutions.

Referring to the ecological fallacy ${ }^{2}$, it has been argued that a job satisfaction-job performance relationship is a hypothesis based on group-level analyses (Latham, 2007; Schneider, 1985). Ostroff (1992) carried out an organizational-level analysis stating that most previous empirical examinations discussed the issue at an individual level and lacked understanding at a group level. However, few empirical examinations have discussed the correlation at both individual and group levels within a single empirical survey. For this reason, we included both individual and group levels in our models to achieve a more comprehensive understanding of the job satisfaction-job performance relationship.

\section{Theoretical background and purpose}

\subsection{Job performance in creative and formulaic tasks of scientists}

Most literature cited in early studies in the 1950s and 1960s on the relationship between job satisfaction and job performance dealt with simple labor (Brayfield \& Crokett, 1955; Latham, 2007; Vroom, 1964). However, current social and economic structures have greatly changed with the advance of science and technology. The quality of jobs is also changing significantly in the rapidly evolving globalized information society. Job complexity has significantly increased in every occupation and greater emphasis is placed on the creativity of each employee. In this context, the importance of examining attitude and behavior in relation to highly skilled professionals is growing in organizational settings. For this study, focusing on scientists as a typical example of highly skilled professionals is effective for making future predictions because scientific research is both highly complex and creative. In particular, this study focuses on scientists engaged in research in the basic sciences at the following five national scientific research institutions in Japan: National Astronomical Observatory of Japan, National Institute for Fusion Science, Institute for Molecular Science, Institute of Space and Astronautical Science and Japan Aerospace Exploration Agency, and the High Energy Accelerator Research Organi- 
zation. These public scientific institutions are recognized globally for being at the forefront in the development of cutting-edge physical science research and advanced scientific devices, including the Subaru Telescope, the Large Helical Device (LHD), and the KEKB accelerator ${ }^{3}$. These national institutions operate many largescale scientific projects and feature scientists' work. Scientists are expected to contribute through group performance in projects as well as individual performance. Current public scientific-research institutions that participate in physical sciences rarely conduct research solely at the entire organization or individual level. In general, they perform research activities at a project or group level. Therefore, from the management point of view, the group level should be the most appropriate and important unit to measure and discuss about achieving outcomes for the organizations investigated in the present study. Using survey data collected from these scientists, this study aims to quantitatively deepen our understanding of the job satisfaction-job performance relationship by measuring the two levels of actors in organizations; i.e., group and individual.

Judge et al. (2001) verified the hypothesis that the correlation between job satisfaction and job performance is higher in high-complexity jobs than in low-complexity jobs by using job complexity as a moderator. They revealed that the highest correlations were observed for scientists-engineers and the lowest were observed for laborers. However, this explanation is incomplete because they found a lower correlation for nurses and accountants than for clerical workers. Furthermore, Argyle (1989) proposed the hypothesis that the correlation was higher in white-collar workers than in blue-collar workers. This hypothesis still cannot explain the distri- butional disparity across occupations observed in Judge et al.'s (2001) investigation. Thus, we proposed that these results should be explained on the basis of creativity and introduced this concept to the current study. In particular, we categorized scientists' job performance into two types: job performance in creative tasks and job performance in formulaic tasks. The former is quantified by the scientists' publication productivity in terms of research articles, patents, and related work and the latter is quantified by publication productivity in terms of reports submitted to governmental offices and related work. As highly skilled professionals, scientists are involved in tasks that are highly complex and require advanced skills. Both types of tasks presented above are complex and require highly skilled professional knowledge; however, the former additionally requires creativity to produce novel knowledge while the latter additionally requires the ability to follow specifications to produce formulaic documents. In an interview conducted as a prestudy for this survey ${ }^{4}$, one scientist described the latter task as "the creation of explanatory materials for bureaucrats" and the "need to produce them in a certain manner." For scientists involved in large-scale projects that require managing large-scale budgets, this reporting of research is an important task. In the field of technology management, creativity is mostly measured by considering patent publication as an indicator. In this study, our attention is focused on scientists; therefore, publications such as research articles are also included as indicators of creativity.

Some organizational researchers have pointed out that achievement without professional growth or improved competence might lead to dissatisfaction (Latham and Yukl, 1976; Latham, 2007; Parker, 2003). The supposition that the 
participants in this study, i.e., scientists, experience the greatest growth and improvement in their competencies when they exercise their creativity and achieve a scientific breakthrough leads to the hypothesis below:

Hypothesis 1: The relationship between job performance and job satisfaction is stronger in creative tasks than in formulaic tasks.

Correlation analyses were conducted based on Hypothesis 1.

\subsection{Measuring job performance}

It is important to recognize that performance indicates different concepts in empirical examinations. Typical examples include the two different concepts of task performance and contextual performance. Motowidlo and Van Scotter (1994) stated that the former is the central task in a workplace. Borman and Motowidlo (1993, 1997) suggested that contextual performance represented a task rather than a support activity for employees to promote the central task. Empirical examinations that define organizational citizenship behavior (Organ, 1988), a concept similar to contextual performance, as performance have been conducted. Some studies have reported that the correlation between job satisfaction and job performance is higher and bidirectional if organizational citizenship behavior is conceptualized as performance (Bateman and Organ, 1983; Schneider, 1985). In this study, however, we define task performance as the central task of scientists.

In most previous empirical examinations, each individual's job performance was measured on the basis of supervisor ratings or peer-subordinate ratings $^{5}$ (e.g., Shore and Martin, 1989). In any case, these ratings are subjective; ratings by a rater are strongly affected by his/her bias.
Another considerable debate involves directly applying task performance scales developed by western cultures to eastern cultures (Fisher and Hartel, 2004; Ng et al., 2009). We try to solve these issues by applying an approach from scientometrics with the number of publications as an objective indicator of the task performance of scientists to confirm whether the job satisfaction -job performance relationship is also observed through this approach.

\subsection{Overall job satisfaction and facet job satisfaction}

According to Locke (1976: 1304), job satisfaction is defined as a "pleasurable or positive emotional state resulting from the appraisal of one's job or job experiences." This is probably the most commonly accepted definition of job satisfaction. However, this definition is now believed to be lacking in a clear distinction between overall job satisfaction (satisfaction with one's job as a whole) and facet job satisfaction (satisfaction with individual matters such as pay as well as specific matters such as a supervisor's management ability). These two types of satisfaction have often been differentiated in recent studies (Shimazu, 2010) ${ }^{6}$. For instance, Judge et al. (2001) has pointed out the drawbacks of applying the average scores of various facet job satisfaction scales as overall job satisfaction and that one should use the overall job-satisfaction scale to appropriately estimate the relationship between overall job satisfaction and job performance. Therefore, in this study, we discuss overall job satisfaction with multiple questionnaire items developed in the Japanese language to construct overall job satisfaction. 


\subsection{Individual-level and group-level correlations}

Two influential reviews of the job satisfaction -job performance relationship have discussed individual-level analysis (Iaffalno and Muchinski, 1985; Judge et al., 2001). Ostroff (1992) pointed out that previous studies on the correlation between the two showed almost no correlation, and that these studies concentrated on individual-level analysis and lacked organization-level analysis. To measure school performance, she examined the correlations between the organization (school)-level average of teachers' job satisfaction and the average of multiple variables such as students' grades and teachers' turnover rate at each school. The results revealed that the school-level job satisfaction of teachers was significantly correlated with school-level performance. A recent meta-analysis by Whitman et al. (2010) discussed the relationship between unit-level collective job satisfaction and job performance and reported a correlation between them. Considering the group-level correlation between job satisfaction and job performance, Latham (2007: 106) stated that "when morale was high, the productivity of the organization was also high' and interpreted its meaning as 'individual-level attitudes and behaviors became shared and produced an emergent collective structure of attitudes, norms, and behaviors that affected organizational outcomes' (Latham, 2007: 107). Should the correlation between job satisfaction and job performance, then, be discussed only at the group level? To confirm that a proposition which holds true in a group does not always hold true for individuals, it is necessary to analyze the same participants at both group and individual levels. To date, few empirical studies have simultaneously explored the job satisfaction-job performance relationship at both levels. This study discusses the relationship between the two at both group and individual levels.

Furthermore, we introduce the level of research activity as a morale-related behavioral variable. In this study, the frequency of the individual behavior of holding scientific workshops or seminars within and outside the organization is measured as an indicator of the level of research activity because these behaviors are directly related to the level of morale associated with scientific research. Similarly, job satisfaction is defined as an attitudinal variable directly related to the level of morale associated with scientific research. The current study discusses in detail the relationship between job satisfaction and job performance by contrasting a morale-related behavioral variable, level of research activity, with a morale-related attitudinal variable, job satisfaction, at both group and individual levels. Like previous studies that used cross-sectional surveys, this study investigates the correlation between the two variables. However, on the basis of social-psychological assumption that attitude precedes behavior and following previous empirical studies (e.g., Borgogni et al., 2010; Fulmer et al., 2003; Harter et al., 2002; Nishii et al., 2008; Ostroff, 1992), we investigated job satisfaction as an explanatory variable and job performance as an explained variable. In particular, we statistically tested the hypothesis below with regression analyses.

Hypothesis 2: Morale-related variables explain higher job performance at both group and individual levels.

This study aims to determine whether job satisfaction-job performance relationship models vary with regard to correlation strength when creativity is considered as a task characteristic. 
Through this examination, we further explore the influence of job satisfaction on job performance at both individual and group levels by contrasting scientists' overall job satisfaction with their level of research activity. Finally, we discuss practical implications and future suggestions derived from the results. Our study applies an interdisciplinary approach using more objective indicators of task performance to provide a clear and more comprehensive reporting of the results. By this approach, our study seeks to better understand human attitudes and behaviors in organizational settings.

\section{Method}

\subsection{Survey instruments}

Questionnaire Questionnaires were distributed to all researchers working at the five Japanese public scientific research institutions. These national institutions are supervised by the Japanese Ministry of Education, Culture, Sports, Science, and Technology.

For distributing the questionnaires, first, in-person visits were made to the leaders of the research groups. Then, the outline of the survey was explained directly by the first author. The leaders were also asked about the number of researchers in their project groups, and accordingly, they received the appropriate number of questionnaires. When a leader was absent on the day of the visit, a secretarial staff member of the group was asked about the number of researchers and the member received the questionnaires. The secretarial staff members were also requested to transfer the e-mails explaining the outline of the survey to all research members in the group. This procedure was necessary to obtain more accurate numbers of researchactive scientists because, sometimes, names of part-time or adjunct scientists do not appear in the list of personnel or on group websites.

The questionnaires were distributed from December 2010 to January 2011. All responses were anonymous. Participation in the study was voluntary, and researchers willing to respond were asked to mail the self-reported responses with a stamped and addressed envelope. A total of 1,240 questionnaires were distributed, of which 494 were returned, giving a response rate of $39.8 \%$. The questionnaires noted that the data would be used for academic research, the survey data would be treated statistically, and individuals would not be identified.

Of the 494 respondents, 457 were male and 37 were female, and the average age was 43 years (43.6 years for men, 35.5 years for women). There were 413 doctoral degree holders, accounting for $83.8 \%$ of the total respondents. Among them, $70.2 \%$ had a degree in science, $22.3 \%$ in engineering, and $7.5 \%$ in other fields.

\subsection{Project groups}

The participants were asked to select their research project group or division. They also indicated their tenure in the group or division. This item was necessary to distinguish between their past and current job performances and measure the correlation between current job satisfaction and current job performance. Fiftyfour groups having three or more respondents were chosen, and we adopted the average values in each group as the group-level variables. The number of group members ranged from 3 to 21 with an average of 8 .

\subsection{Job performance scale}

In this study, we measured scientists' job performance by using the number of publications produced as the objective indicator. We measured publication productivity by consider- 
ing the following seven items partially modified from the UNESCO Survey items (Andrews, 1979), which were translated into Japanese and then revised on the basis of feedback from Japanese scientists at the examined research institutions: (a) books, (b) scientific or technical articles published in refereed journals as a first author, (c) scientific or technical articles published in refereed journals as a coauthor, (d) scientific or technical articles published in refereed conference proceedings as a first author, (e) patents, (f) reports or explanatory materials to be submitted to government and committees, and $(\mathrm{g})$ internal research reports. The variable job performance of creative tasks was measured by calculating the total of each of (a)-(e). Prior to calculating the total for the job performance of creative tasks, (b) scientific or technical articles published in refereed journals as a first author, and (e) patents were weighted double ${ }^{7}$. The variable job performance of formulaic tasks was measured calculating the total (f) plus (g). By excluding articles published without going through refereed systems for (b), (c), and (d), we tried to secure the homogeneity of the measurement in the quality of creativity. In terms of the number of produced publications, for scientists who had worked in the project group for three years or longer, the past three years' performance was measured, whereas for those who had worked for less than three years, the possible performance for three years was calculated using their period of service and the number of publications produced during that period.

\subsection{Job satisfaction scale}

Overall job satisfaction was measured using the four items below, which was partially modified from the Japanese language version of the job satisfaction scale developed by Shimazu
$(2004)^{8}$.

1. Satisfied with current workplace

2. Satisfied with current tasks

3. Satisfied with current duty position

4. My wish is to continue working here for the next five years

Each item was measured on a five-point scale. The sum of the values of the four items was calculated to construct the overall job satisfaction scale. The Cronbach alpha coefficient for the four items was 0.826 , which indicated a sufficient level of reliability (internal consistency) for this scale.

\subsection{Scale of level of research activity}

Few studies have conducted organizational research by constructing scientists' level of research activity with behavioral variables. In the present study, therefore, a new scale for measuring the level of research activity was developed by categorizing the frequency of conducting scientific workshops or seminars as follows: (a) within the project group to which the scientists belong, (b) in the organization to which the scientists belong, or (c) outside the organization. Each was measured on a five-point scale. The Cronbach alpha coefficient for the three items was 0.833 , which indicated a sufficient level of reliability (internal consistency) for this scale.

\section{Results}

To examine Hypothesis 1, we statistically analyzed the correlation between job satisfaction (JS) and both job performance of creative tasks (JP1) and job performance of formulaic tasks (JP2). Table 1 shows the means and standard deviations of each variable and the correlations between the variables at both group and individual levels.

As is shown by the intercorrelations below the diagonal in Table 1, JS has a statistically sig- 
Table 1 Correlation matrix, means and standard deviations of the variables

\begin{tabular}{|c|c|c|c|c|c|c|}
\hline Measure & 1 & 2 & 3 & 4 & $M$ & $S D$ \\
\hline 1. JS & - & 0.083 & $0.419^{* *}$ & 0.040 & 14.541 & 1.566 \\
\hline 2. RA & $0.095^{*}$ & - & $0.446^{* *}$ & 0.071 & 7.150 & 1.225 \\
\hline 3. JP1 & $0.105^{*}$ & $0.264^{* * *}$ & - & 0.100 & 16.709 & 11.205 \\
\hline 4. JP2 & 0.034 & $0.113^{*}$ & $0.260^{* * *}$ & - & 4.771 & 7.219 \\
\hline$M$ & 14.382 & 7.110 & 15.517 & 5.130 & & \\
\hline$S D$ & 3.750 & 2.800 & 19.309 & 17.518 & & \\
\hline
\end{tabular}

Note: Intercorrelations for group level $(n=54)$ and individual level $(n=437)$ are presented above and below the diagonal, respectively. Means and standard deviations for group and individual levels are presented in the vertical columns and the horizontal rows, respectively.

JS = Job satisfaction; RA = Level of research activity; JP1 = Job performance of creative tasks; JP2 = Job performance of formulaic tasks $* * *=p<0.0001, * *=p<0.001, *=p<0.005$

nificant positive correlation with JP1 at an individual level, whereas it has no correlation with JP2. In addition, there is a correlation between JP1 and JP2. Therefore, we can observe that an individual who has a high productivity in JP1 tends to have a high productivity in JP2. Thus, the result of the correlation analysis supports Hypothesis 1. As shown by the intercorrelations above the diagonal in Table 1, JS is also positively correlated with JP1 at the group level, whereas it has no correlation with JP2. The results show that the correlation between JS and JP1 is higher at the group level than at the individual level. Thus, Hypothesis 1 is consistent both in individual and group level analyses. That is, job satisfaction-job performance relationship models vary with regard to correlation strength when creativity is considered as a task characteristic both at individual and group levels.

In response to the results of the correlation analyses at individual and group levels, we set the following working hypotheses and performed regression analyses with ordinary least squares estimation to further understand the role of JS. We compared JS with the variable representing level of research activity (RA) that affects JP. Employment status (permanent or temporary) and age were entered in the regression models as control variables ${ }^{9}$. In this research, JS and RA are defined as the attitudinal and behavioral variables, respectively, directly relating to morale. Furthermore, JS and RA are explanatory variables and JP1 and JP2 are explained variables.

Hypothesis 2: Morale-related variables explain higher job performance at both group and individual levels.

Hypothesis 2-1: Higher JS and RA explain higher JP1 at the individual level.

Hypothesis 2-2: Higher JS and RA explain higher JP1 at the group level.

Hypothesis 2-3: Higher JS and RA explain higher JP2 in individual level.

Hypothesis 2-4: Higher JS and RA explain higher JP2 at the group level.

Results of regression analyses for Hypotheses 2-1 and 2-2 are shown in Table 2 and results of regression analyses for Hypotheses 2-3 and 2-4 are shown in Table 3 .

The individual-level regression model explaining JP1 is significant. Both JS and RA have sig- 
Job satisfaction-job performance relationship for creative tasks

Table 2 Regression analyses for JP1

\begin{tabular}{|c|c|c|c|c|c|c|}
\hline \multirow[b]{3}{*}{ Variable } & \multicolumn{6}{|c|}{ JP1 (Job Performance of Creative Tasks) } \\
\hline & \multicolumn{3}{|c|}{ Individual } & \multicolumn{3}{|c|}{ Group } \\
\hline & B & $95 \% \mathrm{CI}$ & $\beta$ & B & $95 \% \mathrm{CI}$ & $\beta$ \\
\hline Constant & -7.013 & {$[-17.344,3.318]$} & & -44.247 & {$[-77.993,-10.500]$} & \\
\hline JS & $0.486^{*}$ & {$[0.011,0.961]$} & 0.092 & $2.684 * *$ & {$[1.028,4.341]$} & 0.375 \\
\hline RA & $1.879 * * *$ & {$[1.203,2.556]$} & 0.265 & $3.939 * *$ & {$[1.785,6.094]$} & 0.431 \\
\hline$F(d f 1, d f 2)$ & & $10.920 * * *(4,445)$ & & & $9.009 * * * \quad(3,50)$ & \\
\hline$R^{2}$ & & 0.089 & & & 0.351 & \\
\hline Adjusted $R^{2}$ & & 0.081 & & & 0.312 & \\
\hline$n$ & & 450 & & & 54 & \\
\hline
\end{tabular}

Note: Employment status (permanent or temporary) and age are controlled for individual level model and group average age is controlled for group level model

$\mathrm{JS}=$ Job satisfaction; RA = Level of research activity; JP1 = Job performance of creative tasks $* * *=p<0.0001, * *=p<0.001, *=p<0.005$

Table 3 Regression analyses for JP2

\begin{tabular}{|c|c|c|c|c|c|c|}
\hline \multirow[b]{3}{*}{ Variable } & \multicolumn{6}{|c|}{ JP2 (Job Performance of Formulaic Tasks) } \\
\hline & \multicolumn{3}{|c|}{ Individual } & \multicolumn{3}{|c|}{ Group } \\
\hline & $\mathrm{B}$ & $95 \% \mathrm{CI}$ & $\beta$ & B & $95 \% \mathrm{CI}$ & $\beta$ \\
\hline Constant & -2.137 & {$[-9.494,5.219]$} & & 0.876 & {$[-26.019,27.772]$} & \\
\hline JS & 0.077 & {$[-0.355,0.509]$} & 0.017 & 0.142 & {$[-1.178,1.463]$} & 0.031 \\
\hline RA & 0.324 & {$[-0.295,0.943]$} & 0.052 & 0.432 & {$[-1.285,2.149]$} & 0.073 \\
\hline$F(d f 1, d f 2)$ & \multicolumn{3}{|c|}{$4.673 * * \quad(4,433)$} & \multicolumn{3}{|c|}{$0.112(3,50)$} \\
\hline$R^{2}$ & \multicolumn{3}{|c|}{0.041} & \multicolumn{3}{|c|}{0.007} \\
\hline Adjusted $R^{2}$ & \multicolumn{3}{|c|}{0.033} & \multicolumn{3}{|c|}{-0.053} \\
\hline$n$ & \multicolumn{3}{|c|}{438} & \multicolumn{3}{|c|}{54} \\
\hline
\end{tabular}

Note: Employment status (permanent or temporary) and age are controlled for individual level model and group average age is controlled for group level model.

$\mathrm{JS}=$ Job satisfaction; RA = Level of research activity; JP2 = Job performance of formulaic tasks $* * *=p<0.0001, * *=p<0.001, *=p<0.005$

nificant coefficients. RA, with a standardized coefficient of 0.265 , explains JP1 at an individual level relatively stronger than JS, with a standardized coefficient of 0.092 . Both morale-related variables explain JP1 at the individual level. Thus, Hypothesis 2-1 is supported. The grouplevel regression model that explains JP1 is significant. Because the standardized coefficients of JS and RA are 0.375 and 0.431 , respectively, and both are statistically significant, the two moralerelated variables at the group level explain JP1. The adjusted $R^{2}$ in this regression model is 0.312 , which is relatively higher than that of the individual-level model. Thus, Hypothesis 2-2 is supported. Job performance of creative tasks is affected by the morale-related variables of JS and RA at both group and individual levels.

As shown in Table 3, although the individual- 
level model has a statistically significant $R^{2}$, neither of the explanatory variables (JS and RA) have significant coefficients. Although a significant simple correlation coefficient is observed between RA and JP2 in the individuallevel correlation analysis, RA has no effect on JP2 after controlling for employment status and age. Thus, Hypothesis 2-3 is not supported. Consistent with the individual-level analysis, neither JS nor RA has a significant coefficient for JP2 in the group-level regression model. Thus, Hypothesis 2-4 is not supported.

Results from regression analyses indicate that JS and RA as morale-related attitudinal and behavioral variables, respectively, influence JP1 at both the individual and the group level. In contrast, JS and RA have no influence on JP2. These results indicate that morale-related variables affect only the job performance of creative tasks.

\section{Discussion}

\subsection{Discussion on the key findings}

We investigated the job satisfaction-job performance relationship for scientific research as a typical example of a highly complex occupation by dividing scientists' tasks into two variables: creative and formulaic tasks. Our results demonstrate the following. The correlations between JS and JP are observed only for the job performance of creative tasks for the scientists. JS explains productivity in the performance of creative tasks at both the individual and the group level. In contrast, for the job performance of formulaic tasks, JS does not show any correlation at either the individual or the group level. The reverse phenomenon observed in Judge et al.'s (2001) meta-analysis that the correlation between JS and JP was lower in nurses and accountants, who were thought to be engaged in higher-complexity tasks, than clerical workers implied that job complexity was not the only moderator to be considered. The findings of our study may lead to key insights that support this direction. When exploring the job satisfaction-job performance relationship, scientists found greater satisfaction in performing more creative and discretionary tasks than formulaic tasks. Thus, the use of task creativity in our study proved to be advantageous.

This study also demonstrated that the approach of closely focusing on tasks within the same occupation, not only across occupations, was valid. Focusing on bibliometric information was also effective in facilitating job performance quantification and clear task classification.

To facilitate a deeper understanding of the job satisfaction-job performance relationship, examining both individual and group levels simultaneously rather than separately was required, as performed in our study. According to our findings, the correlation between JS and JP was higher at the group level. Furthermore, the individual-level and group-level regression analyses indicated that the influence of JS on JP1 was considerably higher at the group level than at the individual level. This finding is consistent with the observations of researchers focused on group-level analyses (Ostroff, 1992; Whitman, 2010). Given that there is a statistically significant relationship between JS and JP1, even after controlling for other variables and utilizing objective job performance indicators, we can conclude that job satisfaction is related to job performance at the individual level as well. The influence of RA was also stronger at the group level, although it was not as significant as that of JS. When members of the group were highly satisfied with their job and higher level of research activity, JP1 for the entire 
group was high. The inherent assumption of our results is that morale has a strong effect on the job performance of creative tasks, particularly at the group level.

\subsection{Limitations and suggestions for future research}

This study only considered the individual level and a relatively small group level, with 321 team members. In the future, it will be necessary to confirm whether this result is observed not only at individual and group levels but also on a larger scale, that is, at an organizational level.

In terms of task performance measurement, it may be argued that the focal tasks of scientists include not only research but also management and education. Because educational results can also result in the creation of publications, the aspect of education is reflected in JP1 and the aspect of management is reflected in JP2. From these changes, we can conclude that overall task performance was successfully measured with two objective variables. One of the original contributions of this study is in categorizing task performance into two variables based on the concept of creativity. By doing so, our approach was effective in finding another factor that influences the job satisfaction-job performance relationship, i.e., task creativity, apart from the previously reported factors of job complexity and job type. In fact, many scientists would feel uncomfortable with the integration of JP1 and JP2 as one variable. Integration of all tasks of an occupation for measuring overall task performance in one construct may lead to integration of tasks that require differently oriented abilities or efforts. Inconsistent results for the job satisfaction-job performance correlation presented by previous studies may be ascribed to the measurement of job performance used, which possibly integrated tasks that require abilities or efforts with different orientations.

In this study, the approach of focusing on two different tasks is the basis of our assertion that task creativity plays a key role in the job satisfaction-job performance relationship even while controlling for task complexity. However, the validity of our hypothesis that differences in task creativity explain the job satisfaction-job performance relationship difference should be examined in other occupations that have a similar dualistic structure.

The present study demonstrated the necessity and effectiveness of considering both individualand group-level relationships within a single study. From a statistical point of view, building a more comprehensive model that reflects the two-level structure of this data employing multilevel modeling approach (e.g., Hox, 2010) is more appropriate. In that case, factors that are not dealt with in this study should also be incorporated. The relatively small $R^{2}$ of the individual-level regression model presented in the current paper also indicates the existence of other explanatory factors, and these factors would be dealt with in the future researches.

\subsection{Practical implications}

Claiming that scientists including university faculty members find it difficult to find enough time for research because of the administrative burden, the Ministry of Education, Culture, Sports, Science and Technology (MEXT) of Japan is promoting the project of "Building up research administration system" launched from 2011 fiscal year ${ }^{10}$. In this project, MEXT is proposing to facilitate the new positions of "University research administrators (URA)" in universities. For instance, formulaic tasks investigated in 
the present study can be one of the options for URAs taking over the faculty tasks. Our results showed that formulaic tasks did not correlate with scientists' job satisfaction even though they are complex and require highly skilled professional knowledge. Thus, hiring doctoral degree holders with the aptitude for such tasks as URAs should be a measure for expansion of employment opportunities for them as well as facilitating scientific innovation within public scientific research institutions.

Our results, showing that job performance of creative tasks is affected by morale-related variables of job satisfaction and level of research activity, should be quite beneficial to human resource managers who aim to promote scientists' and/or organizational creativity. Managers may benefit from such information and try to increase their employees' satisfaction by emphasizing the likelihood of an improved work environment. A project group leader who applies effective leadership by encouraging employees to increase their level of research activity would also contribute in stimulating scientists' and/or organizational creativity. At the same time, to enhance future competitiveness, promoting researcher creativity is a relatively important theme for not only public institutions engaged in the basic sciences but also for the private sector. Exploring the relationship between task performance and job satisfaction in the same occupation may be particularly important for practitioners interested in creating more immediate productivity gains. We anticipate that this study will mark the beginning of future empirical studies exploring what tasks within occupations are correlated with the overall job satisfaction of employees.

\section{Acknowledgments}

This paper is based on a part of doctoral thesis of the first author (Naoko Kato-Nitta) and revised with the second author (Tadahiko Maeda). The authors thank Nobuo Arimoto, Satoru Ikeuchi, and Yoshio Saito for their assistance in conducting the survey. In addition, they thank Hiroya Hirakimoto, Hideyuki Izumiura, Takashi Nakamura, Koken Ozaki, and Masashi Tachikawa who provided insightful comments and suggestions at the various stages of writing this paper. They would like to express their gratitude to the scientists who responded the questionnaire survey and the public scientific research institutions for their cooperation on this research project. This study is supported by School of Advanced Sciences, the Graduate University for Advanced Studies.

\section{Notes}

1 The method of measuring scientists' performance using the number of scientific or technical articles is used not only in scientometrics but also in the sociology of science, and it is not a new approach in itself. The expression "an approach from scientometrics" refers to the approach of classifying and quantifying job performance using bibliographic information. In other words, we divided various types of publication productivity by scientists into creative tasks and formulaic tasks, which makes this study original.

2 The ecological fallacy, or the ecological inference problem, was originally proposed by Robinson (1950), who noted that correlations observed in aggregated data at the group level are not always observed in individual units of analysis.

3 Subaru Telescope is an 8.2-m optical infrared telescope developed and operated by National Astronomical Observatory of Japan. LHD is a superconducting helical device developed and operated by the National Institute for Fusion Science of Japan. KEKB is a particle accelerator with a ring path approximately $1 \mathrm{~km}$ in diameter. It was developed and is operated by the High Energy Accelerator Research Organization of Japan. Currently, Subaru 
is one of the world's largest optical infrared telescopes, LHD is the world's largest fusion experiment, and KEKB is the world's highest luminosity accelerator

4 The interview was conducted in January 2010 with a Japanese astronomer in his 30s working on a large-scale scientific project.

5 Approximately $89 \%$ of the meta-analysis by Judge et al. (2001) included supervisory or peer ratings.

6 Overall job satisfaction and facet job satisfaction are differentiated in articles that explore the determinants of job satisfaction, whereas in articles that discuss the relationship between job satisfaction and job performance, they are ambiguously differentiated in some cases. As Judge et al. (2001) pointed out in their meta-analysis, Iaffaldano and Muchinsky (1985) measured overall job satisfaction using the average correlation between various job satisfaction factors and job performance.

7 Even in the field of scientometrics or research evaluation, there is no established, reliable, and valid way of weighting the types of publication of scientists to date. However, it appears that a consensus has been established to some extent that the weight of the original papers of the first author is heavier than that of the second author or later. Also, in the field of engineering, obtaining a patent can be considered more important than presenting conference proceedings. Therefore, we doubled the weight of the (b) scientific or technical articles published in refereed journals as a first author, and (e) patents for measuring the job performance and conducted regression analyses. We presented the weighted version of performance indicators in this study; however, we would like to note that the results of regression analyses with the simple summation of (a) through (e) were essentially the same as that of the presented results. In addition, we confirmed that similar results were also obtained with the regression models excluding (a) books. This study does not intend to develop a new performance measure for scientists' evaluation or to argue that the weight adopted in this study should be applied in any context.

8 To lessen the burden on respondents, the number of former questionnaire items of job satisfaction scale developed by Shimazu (2004) was reduced to four items on the basis of the prestudy results.

9 For example, in her study with Australian workers, Aletraris (2010) found that temporary workers report lower levels of job satisfaction.

10 Ministry of Education, Culture, Sports, Science and Technology (MEXT) website, http://www.mext. go.jp/a_menu/jinzai/ura/detail/1315871.htm [in Japanese, accessed in Feb. 2012.]

\section{References}

Aletraris, L. 2010 How satisfied are they and why? A study of job satisfaction, job rewards, gender and temporary agency workers in Australia. Human Relations, 63, 1129-1155.

Andrews, F. M. 1979 Scientific productivity: The effectiveness of research groups in six countries. Cambridge: Cambridge University Press/UNESCO.

Argyle, M. 1989 The Social psychology of work. $2^{\text {nd }}$ edition. Harmondsworth: Penguin.

Bateman, T. S. \& Organ, D. W. 1983 Job satisfaction and the good soldier: The relationship between affect and employee "citizenship". Academy of Management Journal, 26, 887-895.

Borgogni, L., Russo, S. D., Petitta, L. \& Vecchione, M. 2010 Predicting job satisfaction and job performance in a privatized organization. International Public Management Journal, 13, 275-296.

Borman, W. C. \& Motowidlo, S. J. 1993 Expanding the criterion domain to include elements of contextual performance. In N. Schmit \& Borman, W. C. (eds) Personnel selection in organizations. New York: Jossey-Bass, 71-98.

Borman, W. C. \& Motowidlo, S. J. 1997 Task performance and contextual performance: The meaning for personnel selection research. Human Performance, 10, 99-109.

Bowling, N. A. 2007 Is the job satisfaction-job performance relationship spurious? A meta-analytic examination. Journal of Vocational Behavior, 71, 167-185.

Brayfield, A. H. \& Crockett, W. H. 1955 Employee attitudes and employee performance. Psychological Bulletin, 52, 396-494.

Fisher, G. B. \& Hartel, C. E. J. 2004 Evidence for crossvergence in the perception of task and contextual performance: A study of western expatriates working in Thailand. Cross Cultural Management, 11, 3-15.

Fulmer, B., Gerhart, B. \& Scott, K. 2003 Are the 100 best better? An empirical investigation of the relationship between being a "great place to work" and firm performance. Personnel Psychology, 56, 
965-993.

Harter, J. K., Schmidt, F. L. \& Hayes, T. L. 2002 Business-unit-level relationships between employee satisfaction, employee engagement, and business outcomes: A meta-analysis. Journal of Applied Psychology, 87, 268-279.

Hox, J. 2010 Multilevel analysis: Techniques and applications, Second Edition. Routledge Academic.

Iaffaldano, M. T. \& Muchinsky, P. M. 1985 Job satisfaction and job performance: A meta-analysis. Psychological Bulletin, 97, 251-273.

Ivancevic, J. M. 1979 High and low task stimulation jobs: A causal analysis of performance-satisfaction relationships. Academy of Management Journal, 22, 206-222.

Judge, T. A., Bono, J. E., Thoresen, C. J. \& Patton, G. K. 2001 The job satisfaction-job performance relationship: A qualitative and quantitative review. Psychological Bulletin, 127, 376-407.

Latham, G. P. 2007 Work motivation: History, theory, research, and practice. Thousand Oaks, CA: Sage Publications.

Latham, G. P. \& Yukl, G. A. 1976 Effects of assigned and participative goal setting on performance and job satisfaction. Journal of Applied Psychology, 61, 166-171.

Locke, E. A. 1976 The nature and causes of job satisfaction. In M. D. Dunnette (ed) Handbook of industrial and organizational psychology. Chicago: Rand McNally, 1297-1349.

Motowidlo, S. J. \& Van Scotter, J. R. 1994 Evidence that task performance should be distinguished from contextual performance. Journal of Applied Psychology, 79, 475-480.

Ng, T. W. H., Sorensen, K. L. \& Yim, H. K. 2009 Does the job satisfaction-job performance relationship vary across cultures? Journal of Cross-Cultural Psychology, 40, 761-796.

Nishii, L., Lepak, D. \& Schneider, B. 2008 Employee attributions of the "why" of HR practices: Their effects on employee attitudes and behaviors, and customer satisfaction. Personnel Psychology, 61, 503-545.

Organ, D. W. 1988 Organizational citizenship behavior: The good soldier syndrome. Lexington, MA: Lexington Books.

Ostroff, C. 1992 The relationship between satisfaction, attitudes, and performance: An organizational level analysis. Journal of Applied Psychology, 77, 963-974.

Parker, S. K. 2003 Longitudinal effects of lean production on employee outcomes and mediating role of work characteristics. Journal of Applied Psychology, 88, 620-634.

Robinson, W. S. 1950 Ecological correlations and the behavior of individuals. American Sociological Review, 15, 351-357.

Schneider, B. 1985 Organizational behavior. Annual Review of Psychology, 36, 573-611.

Shimazu, M. 2004 Job satisfaction and psychological stress [in Japanese]. Tokyo: Kazama-Shobo.

Shimazu, M. 2010 Shokumu-manzoku to soshiki no kasseika. In Sangyo Soshiki Shinri-gaku Fujimori, T. (ed) Industrial / Organizational Psychology [in Japanese]. Tokyo: Fukumura-Shuppan.

Shore, L. M. \& Martin, H. J. 1989 Job satisfaction and organizational commitment in relation to work performance and turnover intensions. Human Relations, 42, 625-638.

Vroom, V. H. 1964 Work and motivation. New York: John Wiley \& Sons.

Whitman, D. S., Van Rooy, D. L. \& Viswesvaran, C. 2010 Satisfaction, citizenship behaviors, and performance in work units: A meta-analysis of collective construct relations. Personnel Psychology, 63, 41-81.

(Received February 24, 2012 Accepted February 16, 2013) 